\title{
Fabrication of Capacitive Micromachined Ultrasonic Transducers for Underwater Applications using Thermocompression Wafer-Bonder
}

\author{
Mansoor Ahmad ${ }^{1}$, Muhammad Omer Asim ${ }^{2}$ and Ayhan Bozkurt ${ }^{1,3}$ \\ 1 Faculty of Engineering and Natural Sciences, Sabanci University, Istanbul, 34956, Turkey \\ ${ }^{2}$ Centres of Excellence in Science \& Applied Technologies, Islamabad, Pakistan \\ ${ }^{3}$ Sabanci University Nanotechnology Research and Application Center (SUNUM), Istanbul, 34956, Turkey \\ amansoor@ sabanciuniv.edu (M.A), rmoa27@yahoo.com (M.O.A), abozkurt@ sabanciuniv.edu (A.B)
}

\begin{abstract}
Capacitive Micromachined Ultrasonic Transducers (CMUTs) are the prospective alternative to the traditional piezoelectric ultrasonic transducers. CMUTs are essentially parallel plate capacitors produced using Microelectromechanical Systems (MEMS) technology. The production of CMUTs is broken down into sacrificial underetching and wafer bonding methods. The sacrificial release-based techniques are complex and require several adjustments in terms of optimizing fabrication steps and material selections. Further, the sacrificial release-based processes need ultimate control over the gap height and membrane thickness. On the contrary, the wafer bonding fabrication processes are not only simpler than the sacrificial release methods but also provide a very good parametric control over the membrane thickness and gap height. Besides its advantages, the wafer bonding methods are very sensitive to contamination and surface roughness. The surface roughness problems are addressed by either using the costly Silicon-on-Insulator (SOI) wafers or by using complex Chemical Mechanical Polishing (CMP) method. This article presents a simple and economical CMUTs wafer bonding fabrication method. A thermocompression based metal bonded technique is adopted to successfully fabricate low frequency CMUTs to be used for underwater applications.
\end{abstract}

Keywords: Capacitive Micromachined Ultrasonic Transducers (CMUTs); Microelectromechanical Systems (MEMS); Underwater Communication; Wafer Bonding; Thermocompression.

\section{INTRODUCTION}

Capacitive Micromachined Ultrasonic Transducers (CMUTs) introduced in 1994 are the prospective alternatives to piezoelectric transducers [1]. CMUTs were initially introduced to produce ultrasonic transducers for airborne applications [1]. Later, in an underwater experiment researchers revealed that the CMUTs can perform far better in immersion applications due to having a bandwidth advantage over the piezoelectric transducers [2].

A CMUT is basically a parallel plate capacitor consists of a fixed substrate and a flexible membrane suspended over a vacuum cavity. Microelectromechanical System (MEMS) technology is used to fabricate CMUTs [3]. The fabrication methods for CMUTs are further divided into sacrificial release method and wafer-bonding process [1], [4]. In the sacrificialrelease-process the sacrificial layer, patterned on the fixed substrate, describes the cavity height, size and shape of the CMUT. A flexible membrane is added over the sacrificial layer, followed by sacrificial release under-etching step using etch holes to remove the sacrificial layer. The sacrificial release process involves several fabrication and optimization problems. Such as, the alignment steps, the selection of material for the membrane, sacrificial layer, and etchants needs to be optimized. Also, attaining even membrane thickness and underetching a uniform cavity is quite challenging [5], [6]. Furthermore, For low operational frequencies, a comparatively thin membrane with large radius and a uniform vacuum cavity are desired. Contrarily, in devices with large radius, it is difficult for the membrane to withstand the suspension during the sacrificial underetching step. Consequently, the waferbonding method is preferred to adopt to address the cavity uniformity and membrane-stiction issues.

The wafer-bonding process also uses the MEMS technology, but doesn't involve the sacrificial underetching steps. The process require a pair of wafers usually a prime and a siliconon-insulator (SOI) wafer; where the cavity is formed in the prime wafer and then bonded to the SOI wafer which act as the membrane [7]. In the wafer-bonding process, the cavity etched has better uniformity and the thickness of the membrane is determined by the active layer of the wafer which acts as the membrane [8].

Wafer direct bonding, anodic bonding, and adhesive-based are the typical wafer-bonding techniques reported for CMUTs fabrications [9], [10], [11]. The major drawback associated with the wafer bonding is its sensitivity to the contamination and roughness at the bonding surfaces of the wafers [5]. The surface roughness problem can be solved by using the SOI wafers, however the production of the SOI wafers is a complex and high-cost process [4], [5]. Alternatively, a silicon nitride wafer can also be used to replace the SOI wafer, still it requires the complex Chemical Mechanical Polishing (CMP) treatment [12]. Another drawback of the wafer-bonding methods is its high process temperature requirements [12]. Anodicbonding and Adhesive-polymer-based techniques have been reported to ease the surface smoothness and high annealing temperature requirements respectively [9], [11]. However, 